\title{
Optimal Power Flow for a Power System under Particle Swarm Optimization (PSO) based
}

\author{
Vian Hasan Ahgajan \\ Electrical Engineering Department \\ University of Razi
}

\author{
Firas Mohammed Tuaimah, $\mathrm{PhD}$ \\ Electrical Engineering Department \\ University of Baghdad
}

\begin{abstract}
Optimal power flow (OPF) is one of the most vital tool for power system operation analysis, which require complex mathematical formulation to find best solution .particle swarm optimization is one among many methods for solving nonlinear optimization problems and it one of the swarm intelligences. Optimal power flow is one of nonlinear constrained and occasionally combinatorial optimization problems of power system the objective of an optimal power flow is to find steady state operation point which minimizes generation cost, loss, load ability. The OPF solution includes an objective function. A common objective function concerns the active power generation cost. A particle swarm optimization (PSO) is proposed to solve OPF problem. After solving OPF problem the results of PSO would be compered by using many methods such as linear programming, genetic algorithm .The proposed PSO is verified by IEEE-30 in all case studies PSO shown to achieve a lower cost and losses than it when there is line outage and generator outage.
\end{abstract}

Conventional load flow is used to perform the equality constraints. A computer program, written in MATLAB environment, is developed to represent the proposed method.

\section{Keywords}

Optimal power flow, particle swarm optimization, economic dispatch

\section{INTRODUCTION}

In electric power grids, the optimal power flow (OPF) problem is of great importance for power system operators (SO) to maintain a reliable and economic power system operation. The main goals of OPF are to optimize the fuel cost, power losses, voltage stability, and emission cost, while satisfying system constraints. Traditional OPF involving conventional fossil-fuel power plants is a highly nonlinear, nonconvex and mixed integer problem [1] [2]. Optimal Power Flow (OPF) solution methods have been developed over the years to meet this very practical requirement of power system operation [3] [4]

[5]has discussed the optimal power flow problem since its introduction. Because the OPF is a very large, non-linear mathematical programming problem, it has taken decades to develop efficient algorithms for its solution. Many different mathematical techniques have been employed for its solution. the OPF is a non-linear, non-convex optimization problem due to the cost functions and constraints of a large number of power plants integrated into the power grid. A wide range of traditional optimization techniques such as quadratic programming, nonlinear programming, interior point method, mixed integer programming [6] [7] [8]have already been implemented in this field. Some of the techniques have even been adopted by industry because of their fast convergence and robustness. However, those approaches linearize the OPF problem first, and fail to consider the non-smooth, nondifferentiable and non-convex properties. of the system. To circumvent such problem, various modern heuristic optimization algorithms have been developed for power system optimization [9]because such techniques tackle the original problem without modifying it. In general, heuristic algorithms are developed based on two categories, which are single-solution based, and population based approaches. Several examples of single-solution based approach are tabu search and simulated annealing [10] [11]while population based approaches include particle swarm optimization (PSO), gravitational search algorithm (GSA), differential evolution (DE), genetic algorithm (GA), harmony search, and artificial bee colony [12][1] [13][14] [15]. The majority of the techniques discussed in the literature use one of the following five methods [16] [17]

1. Lambda iteration method, also called the equal incremental cost criterion (EICC) method.

2. Gradient method.

3. Newton's method.

4. Linear programming method.

5. Interior point method.

Newly developed heuristic approaches called particle swarm optimization (PSO) has been introduced. This method combines social psychology principles and evolutionary computation to motivate the behaviour of organisms such as fish schooling, bird flocking, etc. Particle swarm optimization, abbreviated as PSO, is based on the behaviour of a colony or swarm of insects, such as ants, termites, bees, and wasps; a flock of birds; or a school of fish. The particle swarm optimization algorithm mimics the behaviour of these social organisms. The word particle denotes, for example, a bee in a colony or a bird in a flock. Each individual or particle in a swarm behaves in a distributed way

using its own intelligence and the collective or group intelligence of the swarm. As such, if one particle discovers a good path to food, the rest of the swarm will also be able to follow the good path instantly even if their location is far away in the swarm. Optimization methods based on swarm intelligence are called behaviourally inspired algorithms as opposed to the genetic algorithms, which are called evolutionbased procedures. The PSO algorithm was originally proposed by Kennedy and Eberhart in 1995 [18]. In this paper many method are dependent particle swarm optimization - based on optimal power flow for generation cost minimization using as control variable the generator active power compared with linear programming, genetic algorithm based on optimal power flow. The proposed PSO, LP, GA based on OPF in order to find the minimum generation cost values. The proposed approach has been examined and tested on IEEE 30 
bus.

At the end of the optimization process, the transmission losses and convergence time of each test systems are comparatively analysed. In addition, the optimal results obtained from the PSO in this study are compared to the results of LP, GA reported in the literature. As a result, PSO shows better performance in terms of finding lower cost values than those in the literature in a shorter time.

\section{OPTIMAL POWER FLOW}

Optimal power flow purposes to optimize a certain objective function, which must be satisfied the system load flow equations and the limits of element operation condition. The optimal condition is executed by modifying the available controls to minimized an objective function subject to specified operation and security desires the OPF problem is formulated as a nonlinear optimization problem with equality and inequality constraints, as shown below [19]:

$\begin{array}{ll}\text { Objective Function } & : \mathrm{f}(\mathrm{x}, \mathrm{u}) \\ \text { Equality Constraints } & : \mathrm{g}(\mathrm{x}, \mathrm{u})=0 \\ \text { Inequality Constraints } & : \mathrm{h}(\mathrm{x}, \mathrm{u}) \leq 0\end{array}$

\subsection{OPF objective function for full cost minimization}

The optimal power flow problem can be described as an optimizations problem is as follows:

$\mathrm{F}\left(\mathrm{P}_{\mathrm{Gi}}\right)=\sum_{\mathrm{i}=1}^{\mathrm{N}_{\mathrm{G}}} a_{i}+\mathrm{b}_{\mathrm{i}} \mathrm{P}_{\mathrm{Gi}}+\mathrm{c}_{\mathrm{i}} \mathrm{P}_{\mathrm{Gi}}^{2}$

Where:

$\mathrm{F}_{\mathrm{i}}\left(\mathrm{P}_{\mathrm{Gi}}\right):$ cost function

$a_{\mathrm{i}}, \mathrm{b}_{\mathrm{i}}, \mathrm{c}_{\mathrm{i}}:$ cost coefficients

\subsection{Limit Conditions}

1) Load Flow Equations

$P_{G i}-P_{L i}-V_{i} \sum_{j=1}^{N} V_{j}\left(G_{i j} \cos \theta_{i j}+B_{i j} \sin \theta_{i j}\right)=0(5)$

$(i=1, \ldots ., N)$

$Q_{G i}-Q_{L i}-V_{i} \sum_{j=1}^{N} V_{j}\left(G_{i j} \sin \theta_{i j}-B_{i j} \cos \theta_{i j}\right)=0(6)$

$(i=1, \ldots ., N)$

2) Generator Limits

$P_{G i}^{\min } \leq P_{G i} \leq P_{G i}^{\max } \quad i=1, \ldots \ldots N_{G}$

$Q_{G i}^{\min } \leq Q_{G i} \leq Q_{G i}^{\max } \quad i=1, \ldots \ldots N_{G}$

$V_{G i}^{\min } \leq V_{G i} \leq V_{G i}^{\max } \quad i=1, \ldots \ldots N_{G}$

$P_{G i}^{\min }$ : Minimum active power of the generator $i^{\text {th }}$

$P_{G i}^{\max }$ : Maximum active power of the generator $i^{\text {th }}$

$Q_{G i}^{m i n}:$ Minimum reactive power of the generator $i^{t h}$

$Q_{G i}^{\max }$ : Maximum reactive power of the generator $i^{t h}$

$V_{G i}^{\min }$ :Minimum voltage value of the generator $i^{t h}$

$V_{G i}^{\max }:$ Maximum voltage value of the generator $i^{t h}$

3) Transformer Limits

$T_{G i}^{\min } \leq T_{G i} \leq T_{G i}^{\max } \quad i=1, \ldots \ldots N_{T}$

$T_{G i}^{\min }$ : Minimum level control of the transformer $i^{t h}$
$T_{G i}^{\max }:$ Maximum level control of the transformer $i^{t h}$

\section{PARTICLE SWARM OPTIMIZATION}

Inspired by the flocking and schooling patterns of birds and fish, Particle Swarm Optimization (PSO) was invented by Russell Eberhart and James Kennedy in 1995. Originally, these two started out developing computer software simulations of birds flocking around food sources, then later realized how well their algorithms worked on optimization problems. Particle Swarm Optimization might sound complicated, but it's really a very simple algorithm. Over a number of iterations, a group of variables have their values adjusted closer to the member whose value is closest to the target at any given moment. Imagine a flock of birds circling over an area where they can smell a hidden source of food. The particles in the search space adjust their location and velocity according to their own experience and the experience of neighbors.

i. The position and velocity vectors of a particle in an $\mathrm{N}$ dimensional search space are expressed in the equations (11) and (12) :

$X_{i}=\left(X_{i 1}, \ldots, X_{\text {in }}\right)$

$V_{i}=\left(V_{i 1}, \ldots ., V_{i n}\right)$

$X_{i i}$ : Position of particle $i^{t h}$ in a search space with n particles

$V_{i i}$ : Velocity of particle $i^{\text {th }}$ in a search space with $\mathrm{n}$ particles

The best position obtained by a particle is expressed as follows:

Pbest $_{i}=\left(X_{i 1}^{\text {best }}, \ldots \ldots, X_{\text {in }}^{\text {best }}\right)$

The particle that has the best position all among the other particles in the population is expressed in equation (14) :

Gbest $_{i}=\left(X_{i 1}^{\text {best }}, \ldots \ldots, X_{\text {in }}^{\text {best }}\right)$

The velocity and position of each particle updated after $(\mathrm{k}+1)$ steps is formulated as follows:

$X_{i}^{(k+1)}=\left(X_{i}^{k}, \ldots, V_{i}^{(k+1)}\right)$

The velocity of $i^{t h}$ individual at $(\mathrm{k}+1)$ iteration is calculated in equation (16):

$V_{i}^{(k+1)}=$

$w V_{i}^{k}+c_{1}$ rand $_{1} x\left(\right.$ Pbest $\left._{i}^{k}-X_{i}^{k}\right)+c_{2}$ rand $_{2} x\left(\right.$ Gbest $_{i}^{k}-$

$\left.X_{i}^{k}\right) \quad(16)$

$k$

$V_{i}^{k} \quad:$ Velocity of particle $i^{t h}$ at iteration $k$

$X_{i}^{k} \quad$ : Position of particle $i^{t h}$ at iteration $k$

$c_{1}$ and $c_{2} \quad:$ Acceleration coefficients

$w \quad:$ Inertia weight parameter

$\operatorname{rand}_{1}$ and $\operatorname{rand}_{2}:$ Random numbers between $[0,1]$

$w$ Inertia weight parameter as a function of $k$ iteration is expressed as follows:

$w(k)=w_{\text {max }}-\left(\frac{w_{\max }-w_{\min }}{\text { Max.iter. }}\right) X_{k}$

At this point Max.Iter and $\mathrm{k}$ are different from each other and indicate the maximum number of iteration and the current number of iteration, respectively. Maximum velocity is expressed as follows [20] 
$V^{\max }=\left(X^{\max }-X^{\min }\right) / N$
$\mathrm{~N} \quad$ : number of intervals

\subsection{Optimal power flow based on PSO algorithm}

In this study, PSO is applied to IEEE 30 bus system to solve optimal power flow problem and it compared with IEEE 30 bus in case of line-outage and generator-outage in order to check it efficiency and the way its work and the difference in cost function, losses in the cases that mentioned. It finds good starting values for initial population before PSO procedure using Newton Raphson Load Flow equations so that it can provide better probability of detecting global optimum .Application of PSO to OPF is shown in Fig (1).

\section{LINEAR PROGRAMMING}

Linear programming is a mathematical tool used to solve the optimization problems, it has the capability to solve linear objective functions and constraints and non-linear objective functions and constraints through linearization and it has the capability to easily handle the inequality constraints where this is one of Linear programming's powerful features [21]. There are several LP techniques that might be used to solve the optimization problems such as the Graphical method, the Standard (Canonical) form solution and the Simplex method, the last one is the most widely used due to speed and simplicity.

\section{GENETIC ALGORITHM}

GAs are general purpose optimization algorithms based on the mechanics of natural selection and genetics. They operate on string structures (chromosomes), typically a concatenated list of binary digits representing a coding of the control parameters (phenotype) of a given problem. Chromosomes themselves are composed of genes. The real value of a control parameter, encoded in a gene, is called an allele [22].

GAs are an attractive alternative to other optimization methods because of their robustness. There are three major differences between GAs and conventional optimization algorithms. First, GAs operate on the encoded string of the problem parameters rather than the actual parameters of the problem. Each string can be thought of as a chromosome that completely describes one candidate solution to the problem. Second, Gas use a population of points rather than a single point in their search. This allows the GA to explore several areas of the search space simultaneously, reducing the probability of finding local optima. Third, GAs do not require any prior knowledge, space limitations, or special properties of the function to be optimized, such as smoothness, convexity, unimodality, or existence of derivatives. They only require the evaluation of the so-called fitness function (FF) to assign a quality value to every solution produced.

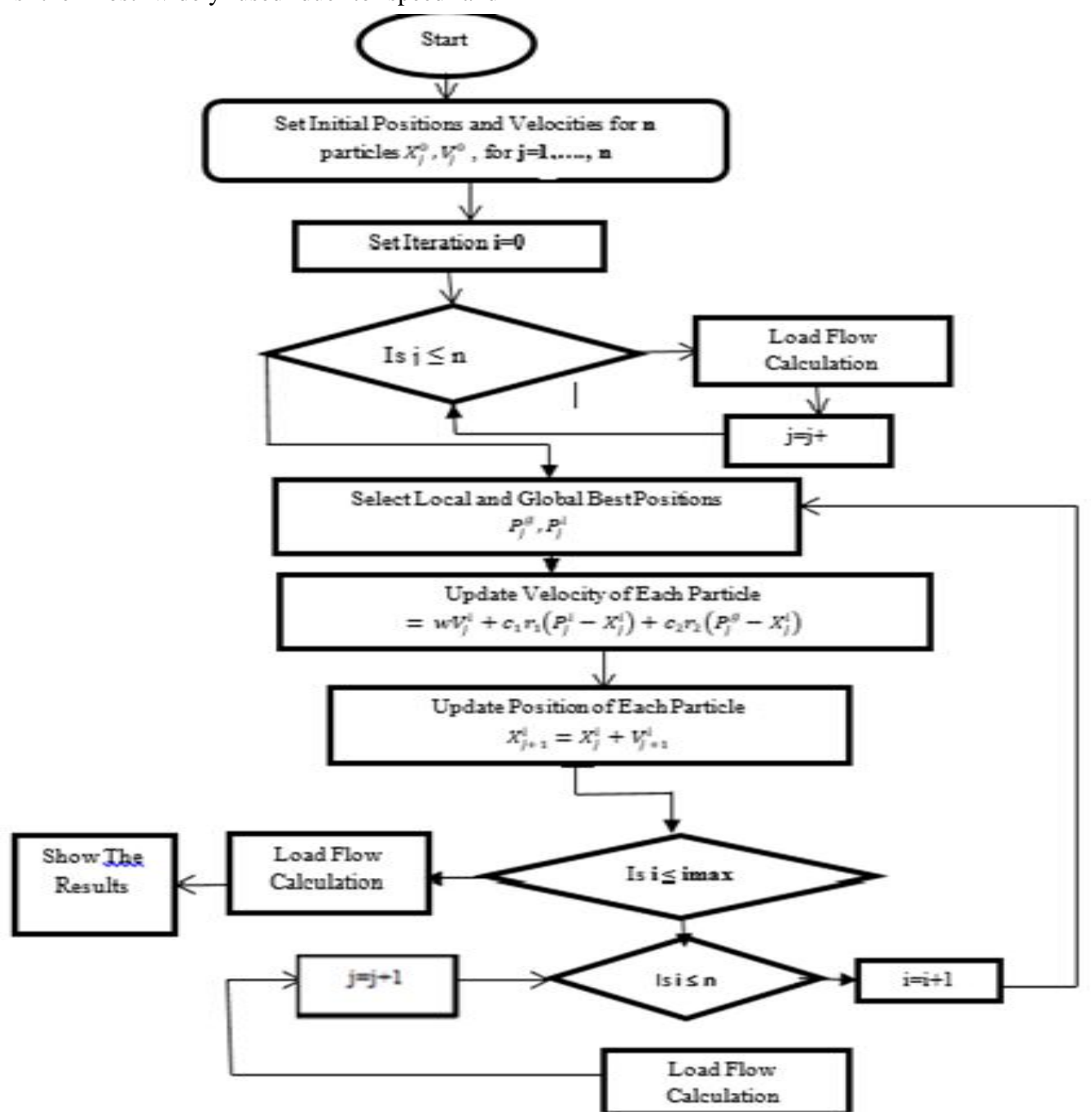

Fig 1: PSO-OPF flow chart 
Table 1. IEEE 30 bus test system generator cos coefficient, Active and reactive power

\begin{tabular}{|c|c|c|c|c|c|c|c|c|c|}
\hline \multicolumn{10}{|c|}{$\begin{array}{c}\text { IEEE } 30 \text { Bus Test System Generator Cost Coefficients, } \\
\text { Active and Reactive Power Outputs }\end{array}$} \\
\hline $\begin{array}{c}\text { Bu } \\
\text { s }\end{array}$ & $\mathbf{a}$ & b & c & e & $\bar{f}$ & $\begin{array}{c}\text { Pm } \\
\text { in }\end{array}$ & $\begin{array}{c}\mathbf{P m} \\
\mathbf{a x}\end{array}$ & $\begin{array}{l}\text { Qmi } \\
\text { n }\end{array}$ & $\begin{array}{l}\text { Qm } \\
\text { ax }\end{array}$ \\
\hline 1 & $\begin{array}{l}0.00 \\
375\end{array}$ & 2 & 0 & 50 & $\begin{array}{c}0.06 \\
3\end{array}$ & 50 & $\begin{array}{c}20 \\
0\end{array}$ & -40 & 200 \\
\hline 2 & $\begin{array}{c}0.01 \\
75\end{array}$ & $\begin{array}{c}1.7 \\
5\end{array}$ & 0 & 40 & $\begin{array}{c}0.09 \\
8\end{array}$ & 20 & 80 & -20 & 100 \\
\hline 5 & $\begin{array}{c}0.06 \\
25\end{array}$ & 1 & 0 & 0 & 0 & 15 & 50 & -15 & 80 \\
\hline 8 & $\begin{array}{l}0.00 \\
834\end{array}$ & $\begin{array}{c}3.2 \\
5\end{array}$ & 0 & 0 & 0 & 10 & 35 & -15 & 60 \\
\hline 11 & $\begin{array}{c}0.02 \\
5\end{array}$ & 3 & 0 & 0 & 0 & 10 & 30 & -10 & 50 \\
\hline 13 & $\begin{array}{c}0.02 \\
5\end{array}$ & 3 & 0 & 0 & 0 & 12 & 40 & -15 & 60 \\
\hline
\end{tabular}

\section{THE SIMULUTION RESULTS}

The proposed PSO-based algorithm for solving OPF problem has been applied to the IEEE 30-bus in this section the numerical results are represented. Loads were modelled as constant powers in the test systems. The results obtained by the proposed approach are compared with the results found by other heuristic methods reported in the literature recently that was on normal condition. After a line outage, generator outage take place in the test system the results of line outage, Generator outage have been compared with the result of the test system under normal condition All the simulations were performed on a personal computer with $3 \mathrm{GHz}$ Intel Processor and $2 \mathrm{~GB}$ of RAM running MATLAB 7.6. Power flow calculations by Newton-Raphson method were performed using the software package MATPOWER.

\subsection{IEEE 30-Bus Test System (normal condition)}

IEEE 30 bus standard test system is shown in Fig 2. The bus and line data of IEEE 30-bus system can be found in [23] while lower and upper limits of the control variables are taken from [24]. The IEEE-30 bus system has six generators at buses1, 2, 5, 8, 11 and 13, and four transformers with off nominal tap ratios at lines 6-9, 6-10, 4-12 and 28-27. In addition, buses $10,12,15,17,20,21,23,24$ and 29 were selected as shunt VAR compensation buses for reactive power control as in [10]. The total system demand was 2.834 p.u. for the active power, and 1.262 p.u. for the reactive power at 100 MVA base. Bus 1 was taken as the slack bus. The generatorbus voltage magnitude limits were assumed 0.95 p.u. and 1.1 p.u., respectively; and the minimum and maximum voltage magnitudes of other buses were considered 0.95 and 1.05 in p.u., respectively. Furthermore, tap settings of the regulating transformers and VAR injections of the shunt capacitors are considered as discrete variables.

The transformer-tap settings were assumed to vary in the range $[0.9,1.1]$ p.u., with step size of 0.0125 p.u [13].The VAR injections of the shunt capacitors

were assumed to vary in the range $[0,0.05]$ p.u., with step size of 0.01 p.u. Fuel cost and emission cost coefficients were taken from [25] [26], respectively. This system has a total of
24 control variables including five generators active power outputs, six generator-bus voltages magnitudes, four transformer-tap settings, and nine shunts VAR injections. Bus test system is shown in figure 2 . The solution of OPF problem with PSO on IEEE 30bus standard test system. The detailed results of objective functions, active and reactive power outputs of generator units, transmission losses of the IEEE 30 bus test system are gathered, explained and compared in TABLE II. Fig 3 show the comparison of the conducted study with similar studies in literature. As a consequence of OPF solutions in the literature with GA, LP, objective functions are found as $804.969,803.89 \$ / \mathrm{h}$, respectively. Although the objective function values with PSO in the literature are found as $801.843 \$ / \mathrm{h}$. transmission losses obtained by GA and LP methods in the literature are 10.29and 4.11 MW. Moreover, the transmission losses with PSO 9.3811MW.fig 4 show the convergence curve for the minimum total fuel cost result from the PSO approach

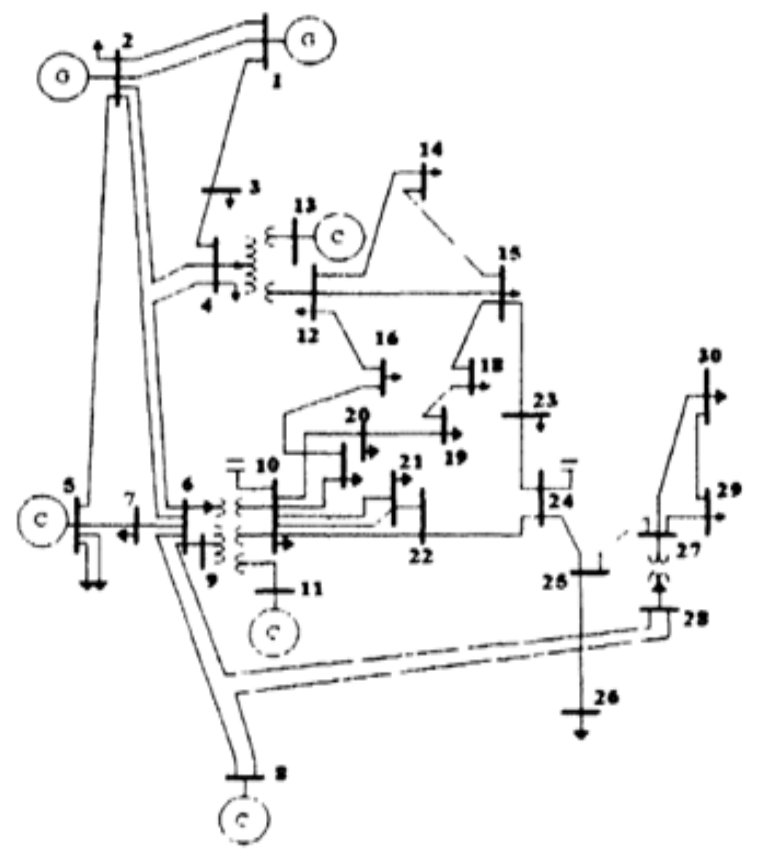

Fig 2. IEEE 30-bus standard test system

It is obvious that the proposed PSO algorithm for this study provide better results than those reported in the literature in terms of total generation cost of the generator units.

Table 2. OPF results for IEEE 30 bus test system

\begin{tabular}{|c|c|c|c|}
\hline IEEE 30-Bus & LP & GA & PSO \\
\hline $\mathrm{P}_{1}(\mathrm{MW})$ & 76.02 & 185.6120 & 176.6907 \\
\hline $\mathrm{P}_{2}(\mathrm{MW})$ & 80.00 & 51.3431 & 48.8354 \\
\hline $\mathrm{P}_{5}(\mathrm{MW})$ & 43.77 & 20.8862 & 21.4639 \\
\hline $\mathrm{P}_{8}(\mathrm{MW})$ & 35.00 & 14.6157 & 21.7001 \\
\hline $\mathrm{P}_{11}(\mathrm{MW})$ & 27.23 & 6.8617 & 12.0831 \\
\hline $\mathrm{P}_{13}(\mathrm{MW})$ & 25.50 & 14.3373 & 12.0000 \\
\hline $\mathrm{P}_{\text {loss }}(\mathrm{MW})$ & 4.114 & 10.296 & 9.3733 \\
\hline Cost $(\$ / \mathrm{h})$ & 803.89 & 804.969 & 801.8436 \\
\hline
\end{tabular}




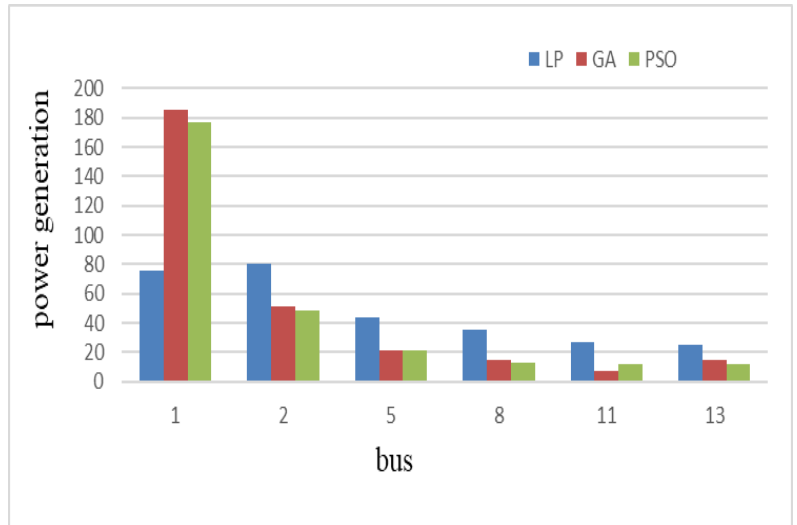

Fig3. Active power generation with normal condition

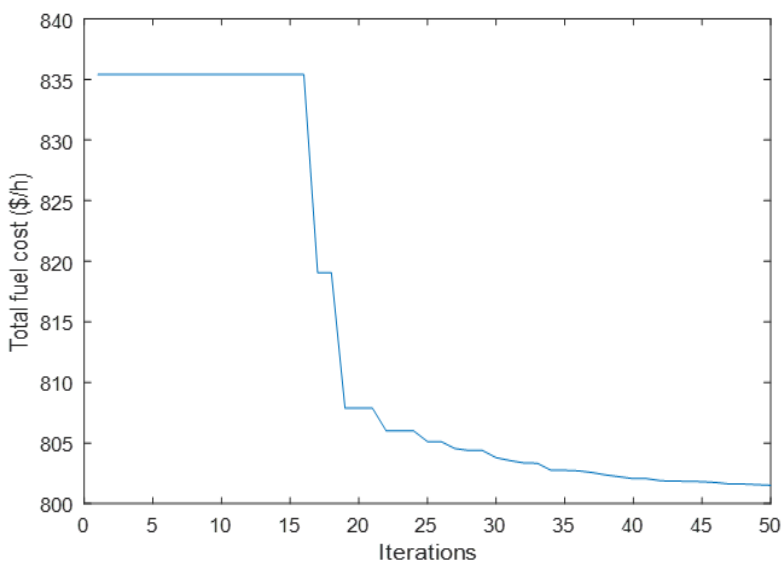

Fig4.The convergence curve for the minimum total fuel cost result from the PSO approach

\subsection{IEEE 30-bus test system (line outage )}

IEEE 30 bus standard test system when a line outage occurred in the system is shown in Fig 5. A line outage occurred in line 6-10,14-15 lead to increase the cost function in the studies case in literature LP,GA and PSO to 804.02, 805.37, 827.3716 $\$ / \mathrm{hr}$ respectively. The detailed results of objective functions, active and reactive power outputs of generator units, transmission losses of the IEEE 30 bus test system are gathered, explained and compared in TABLE III.

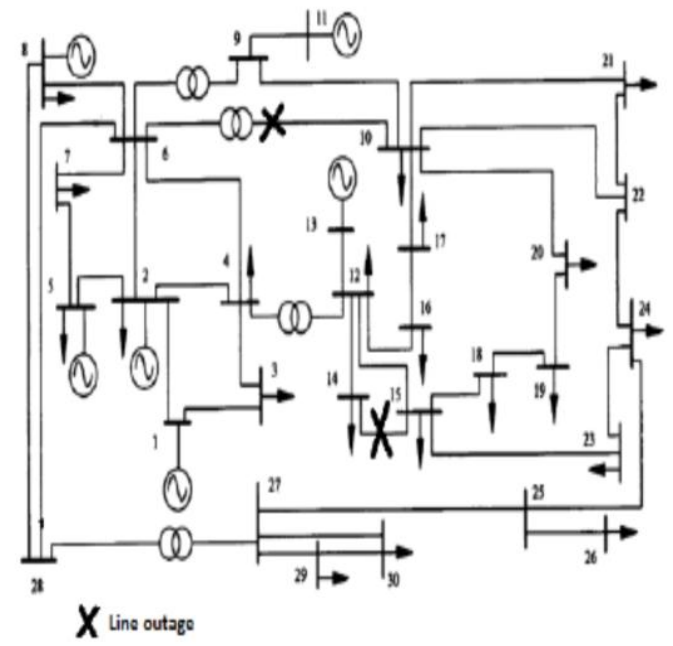

Fig5. IEEE 30 bus standard test system with a line outage in lines 6-10, 14-15
Fig 6 show the convergence curve for the minimum total fuel cost result from the PSO approach when line outage occurred in the system.

Table 3. OPF results for IEEE 30 bus test system ( line outage)

\begin{tabular}{|c|c|c|c|}
\hline IEEE 30-Bus & LP & GA & PSO \\
\hline $\mathrm{P}_{1}(\mathrm{MW})$ & 75.96 & 165.8632 & 157.1444 \\
\hline $\mathrm{P}_{2}(\mathrm{MW})$ & 80.00 & 40.0859 & 41.3853 \\
\hline $\mathrm{P}_{5}(\mathrm{MW})$ & 43.74 & 22.8390 & 19.4483 \\
\hline $\mathrm{P}_{8}(\mathrm{MW})$ & 35.00 & 35.0000 & 10.0000 \\
\hline $\mathrm{P}_{11}(\mathrm{MW})$ & 27.53 & 15.0489 & 28.6438 \\
\hline $\mathrm{P}_{13}(\mathrm{MW})$ & 25.31 & 12.7957 & 34.9316 \\
\hline $\mathrm{P}_{\text {loss }}(\mathrm{MW})$ & 4.114 & 8.2328 & 8.1535 \\
\hline Cost $(\$ / \mathrm{h})$ & 804.02 & 805.8088 & 827.4516 \\
\hline
\end{tabular}

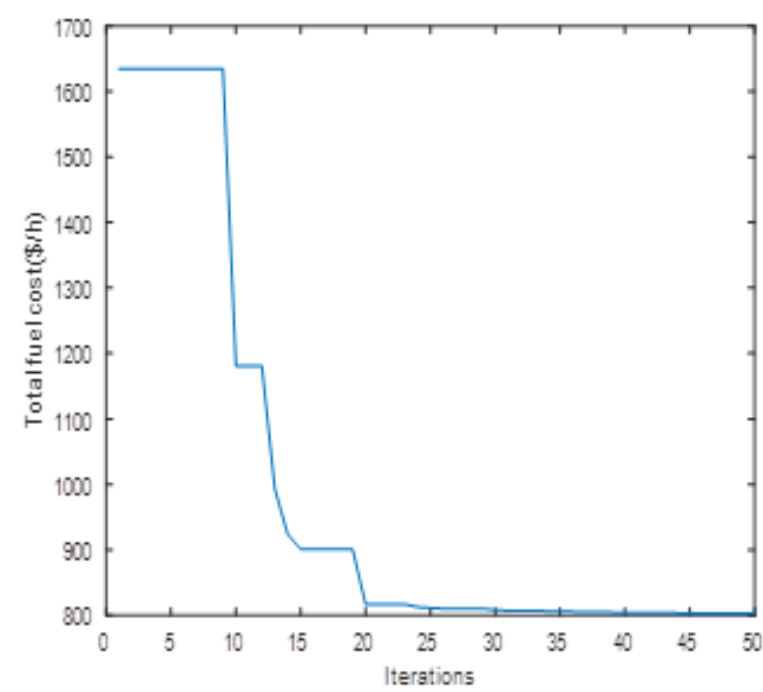

Fig6. The convergence curve for the minimum total fuel cost result from the PSO approach when line outage occurred

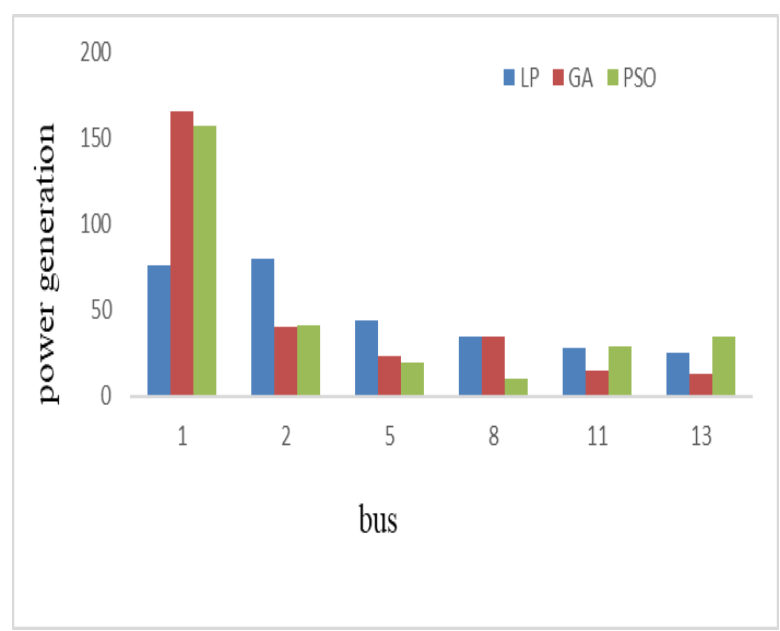

Fig7. Active power generation with line outage 


\subsection{0-bus IEEE test system when it over loaded by $(10 \%, 20 \%$ and $30 \%)$}

Table IV show the results of load flow OPF by using (LP, and PSO) when the system is over loaded by $10 \%, 20 \%$ and $30 \%$

Table 4. OPF results for IEEE 30 bus test system (with over load)

\begin{tabular}{|c|c|c|c|c|c|}
\hline \multicolumn{3}{|c|}{ LP } & \multicolumn{3}{c|}{ PSO } \\
\hline $10 \%$ & $20 \%$ & $30 \%$ & $10 \%$ & $20 \%$ & $30 \%$ \\
\hline 88.51 & 108.12 & 142.79 & 191.14 & 201.75 & 223.02 \\
\hline 80.00 & 80.00 & 80.00 & 50.85 & 54.95 & 61.41 \\
\hline 48.51 & 50.00 & 50.00 & 22.49 & 24.05 & 25.68 \\
\hline 35.00 & 35.00 & 35.00 & 29.98 & 33.30 & 35.00 \\
\hline 30.00 & 30.00 & 30.00 & 14.89 & 16 & 20.56 \\
\hline 36.08 & 40.00 & 40.00 & 13.72 & 16.15 & 17.68 \\
\hline 5.19 & 6.54 & 9.37 & 11.0595 & 13.2779 & 15.8972 \\
\hline 918.03 & 1017.38 & 1173.68 & 907.9789 & 992.176 & $1.1300 \mathrm{e}+03$ \\
\hline
\end{tabular}

Figure 9 show the over load that occurred in 30-bus IEEE test system by using PSO algorithm which about $10 \%$ which cause to increase the cost function and transmission losses in the test system. And figure 10 show the over load that occurred in 30-bus IEEE test system by using PSO algorithm which about $20 \%$ which cause to increase the cost function and transmission losses in the test system.

While figure 11 show the over load that occurred in 30-bus IEEE test system by using PSO algorithm which about $30 \%$ which cause to increase the cost function and transmission losses in the test system

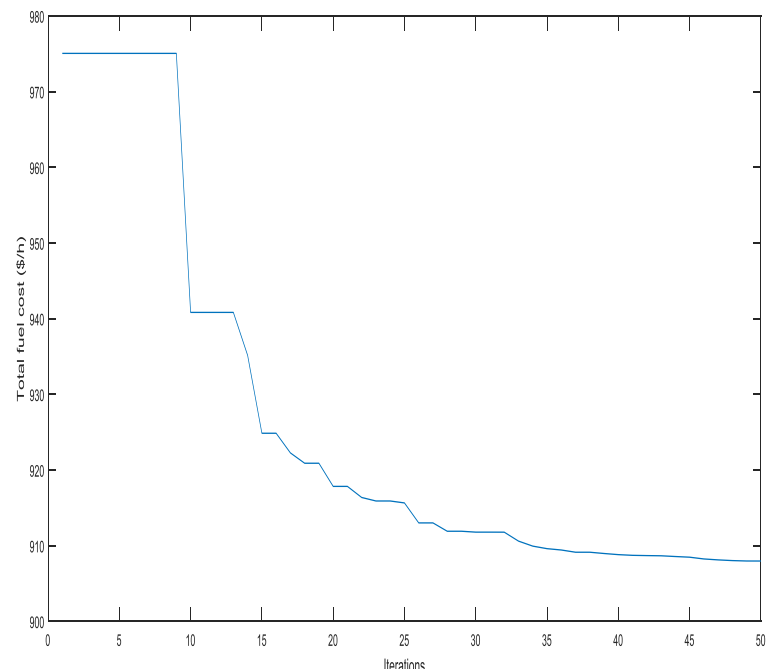

Fig 9. The convergence curve for the minimum total fuel cost result from the PSO Approach with $10 \%$ over load

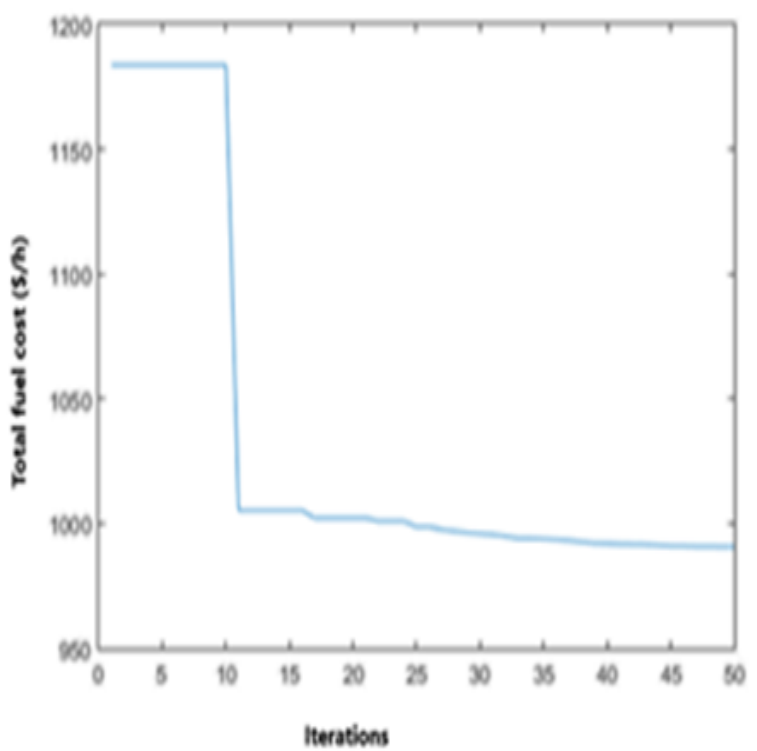

Fig 10. The convergence curve for the minimum total fuel cost result from the PSO Approach with $20 \%$ over load

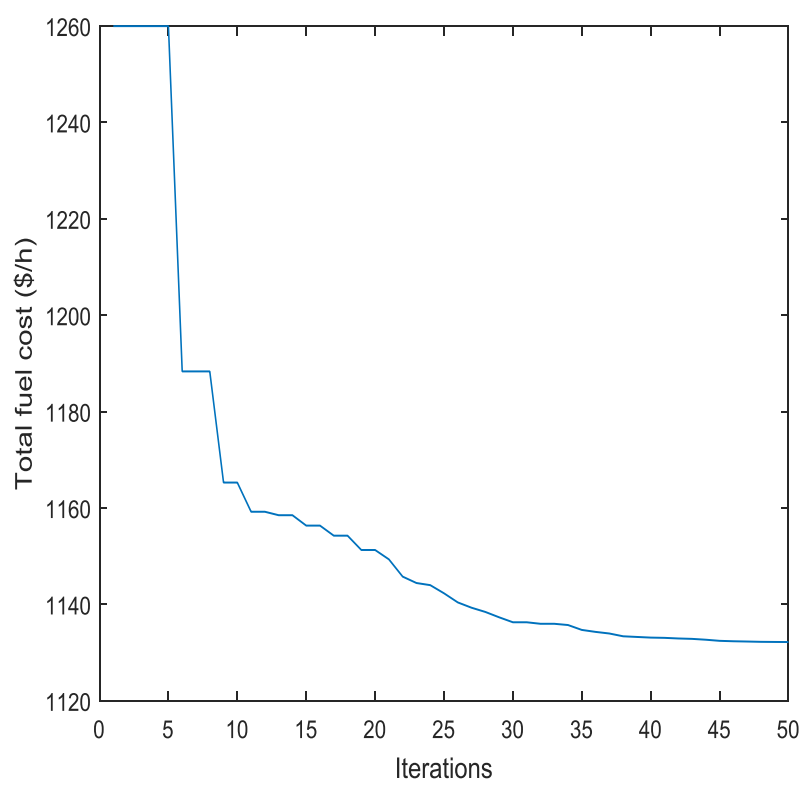

Fig 11. The convergence curve for the minimum total fuel cost result from the PSO Approach with $30 \%$ over load

\section{CONCLUSIONS}

The proposed PSO method developed particularly for this study was displayed a better performance than the OPF methods solved by LP, GA in the literature. The other methods except PSO used in the literature to solve the OPF problem provided more costly objective function values than the PSO method. By using PSO the cost function, transmission losses would be decreased rather than that when LP and GA is used. Even when a contingency takes place in the system it can be noticed that the cost function and transmission losses can be decreased by using PSO algorithm also if the system is loaded (over load) from obtained results in the literature the objective function, losses would be decreased when PSO applied . 


\section{REFERENCES}

[1] M. R. Adaryani and A. Karami, "Artificial bee colony algorithm for solving multi-objective optimal power flow problem," Int. J. Electr. Power Energy Syst., vol. 53, pp. 219-230, 2013.

[2] W. Bai, M. R. Abedi, and K. Y. Lee, "Distributed generation system control strategies with PV and fuel cell in microgrid operation," Control Eng. Pract., vol. 53, pp. 184-193, 2016.

[3] M. E. El-Hawary and D. H. Tsang, "The hydrothermal optimal load flow, a practical formulation and solution techniques using Newton's approach," IEEE Trans. power Syst., vol. 1, no. 3, pp. 157-166, 1986.

[4] E. Acha, H. Ambriz-Perez, and C. R. Fuerte-Esquivel, "Advanced transformer control modeling in an optimal power flow using Newton's method," IEEE Trans. power Syst., vol. 15, no. 1, pp. 290-298, 2000.

[5] J. Carpentier, "Contribution a l'etude du dispatching economique," Bull. la Soc. Fr. des Electr., vol. 3, no. 1, pp. 431-447, 1962.

[6] R. C. Burchett, H. H. Happ, and D. R. Vierath, "Quadratically convergent optimal power flow," IEEE Trans. Power Appar. Syst., no. 11, pp. 3267-3275, 1984.

[7] H. Wei, H. Sasaki, J. Kubokawa, and R. Yokoyama, “An interior point nonlinear programming for optimal power flow problems with a novel data structure," IEEE Trans. Power Syst., vol. 13, no. 3, pp. 870-877, 1998.

[8] R. R. Shoults and D. T. Sun, "Optimal power flow based upon PQ decomposition," IEEE Trans. Power Appar. Syst., no. 2, pp. 397-405, 1982.

[9] K. Y. Lee and M. A. El-Sharkawi, Modern heuristic optimization techniques: theory and applications to power systems, vol. 39. John Wiley \& Sons, 2008.

[10] M. A. Abido, "Optimal power flow using tabu search algorithm," Electr. power components Syst., vol. 30, no. 5, pp. 469-483, 2002.

[11] T. Sousa, J. Soares, Z. A. Vale, H. Morais, and P. Faria, "Simulated annealing metaheuristic to solve the optimal power flow," in Power and Energy Society General Meeting, 2011 IEEE, 2011, pp. 1-8.

[12] A. A. A. El Ela, M. A. Abido, and S. R. Spea, "Optimal power flow using differential evolution algorithm," Electr. Power Syst. Res., vol. 80, no. 7, pp. 878-885, 2010.

[13] A. G. Bakirtzis, P. N. Biskas, C. E. Zoumas, and V. Petridis, "Optimal power flow by enhanced genetic algorithm," IEEE Trans. power Syst., vol. 17, no. 2, pp. 229-236, 2002.
[14] J.-B. Park, Y.-W. Jeong, J.-R. Shin, and K. Y. Lee, “An improved particle swarm optimization for nonconvex economic dispatch problems," IEEE Trans. Power Syst., vol. 25 , no. 1 , pp. 156-166, 2010

[15] S. Sivasubramani and K. S. Swarup, "Multi-objective harmony search algorithm for optimal power flow problem,” Int. J. Electr. Power Energy Syst., vol. 33, no. 3, pp. 745-752, 2011.

[16] A. J. Wood and B. F. Wollenberg, Power generation, operation, and control. John Wiley \& Sons, 2012.

[17] O. Alsac, J. Bright, M. Prais, and B. Stott, "Further developments in LP-based optimal power flow," IEEE Trans. Power Syst., vol. 5, no. 3, pp. 697-711, 1990.

[18] R. Kennedy, "J. and Eberhart, Particle swarm optimization," in Proceedings of IEEE International Conference on Neural Networks IV, pages, 1995, vol. 1000 .

[19] A. J. WOOD and B. Wollenberg, "Power generation operation and control-Second Edition-John Wiley \& Sons.” Inc, 1996.

[20] D. Ben Attous and Y. Labbi, "Particle swarm optimization based optimal power flow for units with non-smooth fuel cost functions," in Electrical and Electronics Engineering, 2009. ELECO 2009. International Conference on, 2009, p. I-377.

[21] K. Zehar and S. Sayah, "Optimal power flow with environmental constraint using a fast successive linear programming algorithm: Application to the algerian power system," Energy Convers. Manag., vol. 49, no. 11, pp. 3362-3366, 2008.

[22] D. E. Goldberg, "Genetic algorithm," Search, Optim. Mach. Learn., pp. 343-349, 1989.

[23] and B. S. Alsac, O., "Optimal load flow with steady-state security," IEEE Trans. power Appar. Syst., no. 3, pp. 745-751, 1974

[24] K. Y. Lee, Y. M. Park, and J. L. Ortiz, "A united approach to optimal real and reactive power dispatch," IEEE Trans. power Appar. Syst., no. 5, pp. 1147-1153, 1985.

[25] S. Duman, U. Güvenç, Y. Sönmez, and N. Yörükeren, "Optimal power flow using gravitational search algorithm," Energy Convers. Manag., vol. 59, pp. 86-95, 2012.

[26] T. Niknam, M. rasoul Narimani, M. Jabbari, and A. R. Malekpour, "A modified shuffle frog leaping algorithm for multi-objective optimal power flow," Energy, vol. 36, no. 11, pp. 6420-6432, 2011. 